\title{
PRÁCTICAS DE LIDERAZGO EN EMPRESAS COMERCIALES EN TAMAULIPAS (MÉXICO)*
}

\author{
NORMA ANGÉLICA PEDRAZA MELO**, JESÚS LAVÍN VERÁSTEGUI***, \\ GERARDO DELGADO RIVAS ${ }^{* * * *}$ \& IDOLINA BERNAL GONZÁLEZ $*$ \\ UNIVERSIDAD AUTÓNOMA DE TAMAULIPAS (MÉXICO)
}

Recibido/ Received/ Recebido: 09/02/2014 - Aceptado/ Accepted / Aprovado: 12/08/2014

\section{Resumen}

\begin{abstract}
El objetivo de este estudio es identificar las prácticas de liderazgo en empresas del sector comercial en Tamaulipas (México). Se aplicó un cuestionario revisado por expertos a una muestra de 84 gerentes. Se usó el análisis factorial exploratorio para identificar la estructura subyacente de las prácticas de liderazgo. Además, los datos fueron analizados con estadística descriptiva, prueba t y ANOVA. Se identificó que las cinco dimensiones del Inventario de Prácticas de Liderazgo (IPL) que están presentes en el ejercicio de liderazgo de la gerencia comercial de las empresas analizadas fueron: desafiar los procesos, inspirar una visión compartida, habilitar a los demás para actuar, modelar el camino y dar aliento al corazón.
\end{abstract}

Palabras clave: Liderazgo, IPL, Prácticas de liderazgo, Comercial, Gerente.

\section{LEADERSHIP PRACTICES IN COMMERCIAL BUSINESS IN TAMAULIPAS (MEXICO)}

\begin{abstract}
The objective of this study is to identify the practices of leadership in business of the commercial sector in Tamaulipas (Mexico). It was applied a questionnaire that was expert-reviewed to a sample of 84 managers. An exploratory factor analysis was used to identify the structure subjacent to the practices of leadership. Besides, the data was analyzed with descriptive statistics, $t$ test and ANOVA. It was identified that the five dimensions of Leadership Practices Inventory (IPL), that are present on the exercise of the commercial management leadership on the companies analyzed are: challenge the process, inspire a shared vision, empower others to act, to model the path and give encouragement to the heart.
\end{abstract}

Keywords: Leadership, IPL, Leadership practices, Commercial, Manager.

* El artículo de investigación correspondiente al área de Administración de Empresas. Es producto del proyecto de investigación: "El liderazgo gerencial en empresas del Estado de Tamaulipas", financiado por la Universidad Autónoma de Tamaulipas, desarrollado entre julio de 2013 y mayo 2014.

** Doctora en Administración por la Universidad Autónoma de Tamaulipas. Miembro del Sistema Nacional de Investigadores en México. Profesora investigadora del Centro de Innovación y Transferencia del Conocimiento (CINOTAM) de la Universidad Autónoma de Tamaulipas. Adscrita al cuerpo académico Gestión Pública y Empresarial de la UAT. Correo electrónico: napedraza@uat.edu.mx, angelicapedrazamelo@gmail.com. Tel: 018343181800 Ext. 2493. Dirección postal: Centro Universitario Adolfo López Mateos, Centro de Innovación y Transferencia del Conocimiento. CP 87149. Ciudad Victoria, Tamaulipas, México.

**** Doctor en Ciencias Administrativas por la Universidad Nacional Autónoma de México. Miembro del Sistema Nacional de Investigadores en México. Adscrito al cuerpo académico Gestión Pública y Empresarial de la UAT. Director del Centro de Innovación y Transferencia del Conocimiento (CINOTAM) de la Universidad Autónoma de Tamaulipas. Correo electrónico: jelavin@uat.edu.mx

***:* Doctor en Ciencias Administrativas por la Universidad Autónoma de Tamaulipas. Profesor investigador adscrito al cuerpo académico Gestión Pública y Empresarial de la UAT. Correo electrónico: jdelgador@uat.edu.mx

******* Estudiante de Doctorado en Ciencias Administrativas en la Universidad Autónoma de Tamaulipas. Profesor de asignatura en la Facultad de Comercio y Administración de la UAT en Ciudad Victoria, Tamaulipas. Correo electrónico: ibernal@uat.edu.mx 


\title{
PRÁTICAS DE LIDERANÇA EM EMPRESAS COMERCIAIS EM TAMAULIPAS (MÉXICO)
}

\begin{abstract}
Resumo
O objetivo deste estudo é identificar as práticas de liderança em empresas do setor comercial em Tamaulipas (México). Aplicou-se um questionário revisado por peritos a uma mostra de 84 gerentes. Usou-se a análise fatorial exploratória para identificar a estrutura subjacente das práticas de liderança. Além do mais, os dados foram analisados com estatística descritiva, prova t e ANOVA. Identificou-se que as cinco dimensões do Inventário de Práticas de Liderança (IPL) que estão presentes no exercício de liderança da gerencia comercial das empresas analisadas foram: desafiar os processos, inspirar uma visão compartilhada, habilitar aos demais para atuar, modelar o caminho $e$ dar fôlego ao coração.
\end{abstract}

Palavras chave: Liderança, IPL, Práticas de liderança, Comercial, Gerente.

Pedraza, N., Lavín, J., Delgado, G. \& Bernal, I. (2015) Prácticas de liderazgo en empresas comerciales en Tamaulipas (México). En: Revista de la Facultad de Ciencias Económicas de la Universidad Militar Nueva Granada. rev.fac.cienc.econ, XXIII (1).

JEL: M12, L25.

\section{Introducción}

De forma cotidiana las empresas, independientemente de su tamaño o actividad económica, se enfrentan a una serie de desafíos para tratar de mantenerse en el mercado. En particular, si referimos a las pequeñas y medianas empresas (PyMES), representan la principal fuente de empleo en la mayoría de las naciones; sin embargo, el entorno en el que se lleva a cabo la creación y desarrollo de estas organizaciones, normalmente se caracteriza por la incertidumbre, por la constante del cambio y la innovación, la inestabilidad política y económica, y sin lugar a dudas por la cultura que prevalece en sus naciones o regiones, esto complica o imposibilita la gestión de las mismas bajo el enfoque de planificación a mediano y largo plazo (Arroyo, 2005).

Normalmente estas entidades manifiestan una serie de errores comunes que terminan con su vida (CONDUSEF, 2013), entre los que se identifican están la ausencia de una cultura empresarial, la falta de análisis estratégico, la mala administración, la incompetencia personal, la mala previsión financiera, adquirir deuda sin previsión, la centralización del poder, la ausencia de controles y la falta de planea- ción. Martínez (2006) menciona que precisamente las habilidades gerenciales pueden afectar la actitud de desarrollo exportador en las PyMES, que implica entre otros factores una falta de interés e imaginación en los ejecutivos de estas organizaciones; considerable preocupación en los problemas del día a día; hombres poco ambiciosos, precavidos y ocupados; así como una actitud pasiva de los directivos. Se estima que esta problemática no solo se presenta en la visión de exportación, se considera que la capacidad del liderazgo efectivo en la gerencia, es necesaria para encausar de manera efectiva y racional el capital humano hacia la consecución de metas organizacionales que permitan a una empresa subsistir competitivamente en su industria.

De acuerdo con Guaipatin (2003), las micro, pequeñas y medianas empresas (MiPyMES) tienen una importante contribución en el crecimiento económico, en la competitividad e innovación, así como en la generación de empleos de los países de América Latina y el Caribe. En particular, se menciona que en Latino América el $99 \%$ de las empresas son MiPyMES, las cuales generan del $48 \%$ al $94 \%$ de los empleos (Guaipatin, 2003). De allí que representan un objeto de estudio de permanente interés en diferentes ac- 
tores: gobierno, empresas privadas, organizaciones no gubernamentales $e$ instituciones de educación superior. En México, estas unidades económicas son parte importante de la economía del país y la principal fuente de empleo. De acuerdo con el Instituto Nacional de Estadística y Geografía (INEGI) en su censo económico del 2009, señala que existían alrededor de 4.5 millones de empresas en esta nación, de las cuales el 99,8\% corresponde a esta categorización, que generan el $52 \%$ del Producto Interno Bruto (PIB) y alrededor del $73 \%$ del empleo nacional. Al situarnos en el panorama del estado de Tamaulipas para el año 2009, existían 269 mil empresas, de las cuales el $99 \%$ son MiPyMES, aportando el $52 \%$ del PIB y $42,1 \%$ del empleo generado (INEGI, 2010).

Con estas cifras descritas, se infiere la relevancia económica de las PyMES, representando un campo de investigación de permanente interés desde diversas perspectivas, en particular la forma en que estas entidades económicas son administradas por sus líderes. Por lo que se estableció la siguiente pregunta de investigación para este trabajo: ¿Cuáles son las prácticas de liderazgo que ejercen los gerentes comerciales de pequeñas y medianas empresas localizadas en Tamaulipas, México? Este cuestionamiento es importante, porque las circunstancias que día a día tienen que hacer frente los gerentes en temas como la globalización, la incertidumbre, la innovación tecnológica, la competitividad internacional intensa, las condiciones económicas y políticas que prevalecen en las naciones donde están ubicadas las empresas, el trabajo en equipo, la productividad empresarial, solo por mencionar algunos factores que inciden en el desempeño y permanencia de las organizaciones, justifica la pertinencia de estudiar el liderazgo empresarial.

De allí que esta investigación, tiene como objetivo general analizar e identificar las prácticas de liderazgo que llevan a cabo los gerentes de empresas pequeñas y medianas del sector comercial en Tamaulipas, México. Para lograr este cometido, se toma como referencia el modelo de Inventario de Prácticas de Liderazgo (IPL) de Kouzes \& Posner (1997, 2003). Se establecen dos objetivos específicos de indagación: i) Identificar las prácticas de liderazgo que realizan los gerentes comerciales de las empresas analizadas y ii) Determinar si existen diferencias entre las prácticas de liderazgo que ejercen los gerentes comerciales respecto a variables demográficas como sexo, edad, estado civil, nivel de escolaridad y una variable organizacional relacionada con el origen de la empresa. De acuerdo con Senge (2006), existen tres factores clave que han impactado y mantienen un dinamismo constante de nuestro mundo, siendo estos: la sociedad del conocimiento, la colaboración masiva y el ritmo acelerado de los cambios; por lo que estudiar el fenómeno del liderazgo se justifica como tema vigente en la agenda de investigación, puesto que los gerentes o líderes empresariales tienen en sus manos la gran responsabilidad del éxito o fracaso de sus organizaciones.

Este trabajo documenta los resultados de investigación realizada en el año 2013. Se presenta la información en cinco apartados. Primeramente se desarrolla una introducción, que describe el contexto empresarial de análisis, la problemática que justifica la pertinencia del estudio, así como la pregunta y objetivos de indagación. En la sección dos se discute información relacionada con el fenómeno de liderazgo, su concepto, importancia y el modelo IPL de Kouzes \& Posner $(1997,2003)$, que ha sido adoptado como base en este estudio. Luego se describe la sección de metodología, que desarrolla el diseño de la investigación y la inmersión al trabajo de campo. Posteriormente, se expone el análisis de los datos e interpretación de los resultados que caracterizan a las prácticas de liderazgo de empresas comerciales localizadas en Tamaulipas, México; finalmente se exponen las principales conclusiones e implicaciones para futuros estudios.

\section{El liderazgo en la gestión empresarial y el modelo IPL}

Las empresas para lograr sus objetivos necesariamente requieren de la cooperación de los empleados, en este sentido el liderazgo de la gerencia representa un factor clave para que el personal se identifique y comprometa en la consecución de los resultado organizacionales y en la eficacia empresarial (Pedraja, Rodríguez \& Rodríguez, 2008; Cuadra \& Veloso, 2009; Rodríguez, 2010; Barahona, Cabrera \& Torres, 2011), fundamentalmente cuando se tiene 
el interés de la longevidad de la empresa (Gómez, Betancourt \& Zapata, 2012).

El liderazgo es una variable de interés en las habilidades directivas. En este sentido, la Escuela de Relaciones Humanas destaca el papel de líder como un facilitador de prácticas de cooperación organizacional, que contribuye al logro de las metas empresariales, y en paralelo se ocupa de satisfacer las necesidades del individuo; sin embargo, es importante tener presente que el liderazgo es un fenómeno que no solo depende directamente de las personas que lo ejercen, también está influenciado por el medio en que se desenvuelva y de la organización en que se desempeña (Barahona, Cabrera \& Torres, 2011). En este marco de ideas sobre la eficacia del líder, se señala de acuerdo con Fiedler (1967), que depende de la situación esta efectividad, de hecho él concluyó en sus estudios, que los líderes enfocados fundamentalmente en la tarea o el trabajo, son más eficaces tanto en situaciones favorables como desfavorables.

Se observa en la literatura que el liderazgo es una variable que ha sido frecuentemente estudiada tanto en el contexto internacional como nacional, así mismo en distintas organizaciones y bajo diversas perspectivas de análisis (Cuadrado, Molero \& Navas, 2003; Pedraja \& Rodríguez, 2004; Cortés, 2004; Mendoza \& Ortiz, 2006; Robles, De la Garza \& Medina, 2008; Robles, 2008; Pedraja, Rodríguez \& Rodríguez, 2008; Cuadra \& Veloso, 2009; Pedraja, Rodríguez, Barredo, Sagredo \& Segovia, 2009; Molero, Recio \& Cuadrado, 2010; Arredondo, 2011; Zarate \& Matviuk, 2012; Contreras \& Juárez, 2013; Contreras \& Castro, 2013; por mencionar algunos estudios que han abordado su análisis).

Por ejemplo, uno de los enfoques de estudio ha relacionado al género y el liderazgo, abordándose el análisis en ámbitos como la educación (Estebaranz \& Mingorance, 2005; Ramos, 2005; Cáceres, Trujillo, Hinojo, Aznar \& García, 2012) y el área empresarial (Contreras, Pedraza \& Mejía, 2012). Resulta de importancia este fenómeno, ya que dentro de los aspectos a valorar cuando se estudia el liderazgo, se considera precisamente la cuestión del sexo, puesto que se ha determinado en estudios previos, que la presencia masculina es mayoritaria en los puestos gerenciales. Sin embargo, se estima que la dirección ejercida por la mujer se vincula mayormente con un estilo de liderazgo transformacional, considerándose más adecuado a las demandas y contingencias que enfrentan las organizaciones en los tiempos actuales, puesto que las mujeres tienen cualidades (capacidad de estimular la cooperación y construcción de equipos, capacidad para ayudar a la gente personal y profesionalmente, entre otros rasgos) que evidencía la importancia de la mujer en puestos directivos de las organizaciones (Estebaranz \& Mingorance, 2005; Ramos, 2005; Cáceres, Trujillo, Hinojo, Aznar \& García, 2012).

Al hablar de liderazgo existe cierto consenso en el transcurso de los años sobre su concepto, que fundamentalmente hace referencia al proceso de influir en los individuos, despertando el interés genuino, responsable, entusiasta y comprometido de contribuir al logro de resultados organizacionales de manera efectiva a la vez que atienden sus necesidades individuales (Amorós, 2007). El liderazgo es una variable necesaria para crear y fortalecer una arquitectura social, que sea capaz de impulsar la realización total de los empleados así como la eficacia organizacional en un entorno de adaptación y preparación para el cambio y la innovación (Contreras \& Castro, 2013). En este contexto, se considera que las cualidades clave para un líder efectivo es ser visionario, capaz de que otros lo sigan, comunicador efectivo, orientado a los resultados, promotor del trabajo en equipo, con capacidad de manejar el cambio, así como de potenciar las capacidades de los trabajadores con base en sus competencias (Wright, 2009; Contreras \& Castro, 2013).

De acuerdo con Barahona, Cabrera \& Torres (2011) en la literatura tiende a mencionarse de manera tradicional a tres estilos de liderazgo, siendo estos el autoritario o autocrático, el democrático y el conocido como laissez-faire (Ramos, 2005). El estilo autoritario se caracteriza porque el jefe o superior toma las decisiones en la organización, sin incentivar a los subalternos en el proceso, supervisa el desempeño y da órdenes de cómo hacer el trabajo; en contraste, el líder democrático estimula la participación del personal al tomar decisiones, les guía y da facultades para llevar a cabo sus tareas; mientras que el laissez-faire, 
se categoriza porque el superior no ejerce influencia en sus subordinados, los deja libremente, se denomina también como no liderazgo.

De acuerdo con Likert (1961), se identifican dos tipos de directivos, los centrados en el empleado, que se ocupan de atender los aspectos humanos de los problemas del personal y de conformar grupos de trabajo eficientes, que les permitan lograr metas de desempeño elevadas, en este sentido se habla del tipo de líder democrático; en contraste están los jefes que se centran fundamentalmente en el trabajo o tarea, caracterizándose este estilo de dirección por obtener un bajo rendimiento en su área, altamente ocupados por definir y clarificar los roles y tareas, identificando al líder autocrático o autoritario como este tipo de jefe o supervisor.

En la década de los setentas se introduce el concepto de líder transaccional y transformacional (Burns, 1978). En esta clasificación Bass $(1985,1990)$ refiere que el liderazgo transaccional se caracteriza porque el superior (gerente, supervisor, director) reconoce las necesidades o logros que pretende el trabajador y se compromete a ayudarlos a conseguir esos resultados a cambio de una compensación u otro tipo de recompensa por su desempeño (acuerdo de intercambio); mientras que el líder transformacional sensibiliza o crea conciencia en el personal sobre la importancia de la consecución de los resultados por su propio interés, los motiva, guía, apoya y manifiesta interés por el desarrollo del individuo dentro de la organización.

De acuerdo con Aronson (2001), las teorías transaccionales identificaban al liderazgo como parte de la supervisión de un grupo de personas, mientras que las transformacionales vendrían a superar al liderazgo transaccional, porque se caracterizan primordialmente por contribuir en la transformación de individuos y organizaciones. El líder transaccional está más enfocado en el intercambio y acuerdo de motivaciones extrínsecas, a controlar las acciones de los colaboradores para obtener su máximo rendimiento, corresponde a un liderazgo técnico y apropiado para empleados inmaduros y que necesitan ser constantemente supervisados (Arredondo, 2011). En tanto que el liderazgo transformacional, se caracteriza por ser innovador y creativo, influye en la conducta de sus subordinados para movilizarlos hacia necesidades de orden superior (Bass, 1998, 1999).

Para analizar el liderazgo en el presente estudio, se fundamenta la investigación en el modelo de Inventario de Prácticas de Liderazgo (IPL) de Kouzes \& Posner (1997, 2003), quienes integran la tipología transaccional y transformacional en las cinco dimensiones que conforman su modelo y que de manera general se presentan en la Tabla 1. Lo anterior con fundamento en Bass \& Riggio (2006), quienes señalan que el instrumento para medir el comportamiento de líderes transformacionales y para desarrollar programas de liderazgo precisamente es el IPL. De allí que con fundamento en ellos se decide abordar el análisis del liderazgo de la gerencia comercial en Tamaulipas, México, sobre la fundamentación del modelo de Kouzes \& Posner (1997, 2003).

El liderazgo gerencial se estima una variable importante que se relaciona tradicionalmente con el desempeño de las organizaciones (Boerner, Eisenbeiss \& Griesser, 2007). Al respecto, Rodríguez (2010) señala que en la literatura se ha identificado a la cultura organizacional y los estilos de liderazgo, como dos variables de las áreas de economía de empresa y del comportamiento organizacional, que se ha vinculado de manera directa e independiente con la eficacia organizacional, y además menciona que la relación entre estilos de liderazgo y eficacia de las organizaciones está ampliamente demostrada. De allí el interés y pertinencia de dar continuidad en el análisis del fenómeno del liderazgo, en particular en empresas comerciales en México, puesto que son las organizaciones con mayor participación en la vida económica de esta nación, representan el 47,1\% del total de las entidades económicas; forman parte del sector terciario que se caracteriza precisamente por aportar el 59,85\% del PIB en este país (INEGI, 2010) y además tienen el 30,5\% del personal ocupado (empleo).

Por lo que es necesario contribuir desde la academia en potenciar la efectividad del liderazgo que ejerce la gerencia de estas empresas, a fin de que puedan permanecer en su mercado. Velázquez (2005) encontró en el análisis de 147 organizaciones mexicanas, que 
Tabla 1. Caracterización de las dimensiones del modelo IPL

\begin{tabular}{|c|c|c|}
\hline Prácticas de liderazgo & Descripción del alcance & Dimensión del liderazgo \\
\hline 1. Desafiar los procesos & $\begin{array}{l}\text { Salir a la búsqueda de oportunidades que impliquen el desafío de } \\
\text { cambiar y crecer, innovar y mejorar. Experimentar, correr riesgos, } \\
\text { extender los experimentos y cuestionamientos asumidos por el } \\
\text { líder. }\end{array}$ & \multirow{4}{*}{$\begin{array}{l}\text { Transformacional: ofrecen una visión y se enfocan en } \\
\text { necesidades internas de orden mayor en los empleados; } \\
\text { incrementando el compromiso de ellos con la visión de } \\
\text { la empresa, mediante la calidad de las relaciones inter- } \\
\text { personales. }\end{array}$} \\
\hline $\begin{array}{l}\text { 2. Inspirar una visión compar- } \\
\text { tida }\end{array}$ & $\begin{array}{l}\text { Implica que el líder imagina un futuro edificante, una visión exci- } \\
\text { tante. Tiene la capacidad de reunir a otros en torno a una visión } \\
\text { común, apelando a sus valores, intereses, esperanzas y sueños. }\end{array}$ & \\
\hline $\begin{array}{l}\text { 3. Habilitar a los demás para } \\
\text { que actúen }\end{array}$ & $\begin{array}{l}\text { Fomentar el trabajo colaborativo y participativo en la toma de de- } \\
\text { cisiones, la generación de confianza. }\end{array}$ & \\
\hline 4. Modelar el camino & $\begin{array}{l}\text { Dar ejemplo de comportamiento coherente con los valores adop- } \\
\text { tados. }\end{array}$ & \\
\hline 5. Dar aliento al corazón & $\begin{array}{l}\text { El líder reconoce las contribuciones individuales al éxito, propor- } \\
\text { ciona retroalimentación positiva y reconocimiento público, cele- } \\
\text { bra los logros del equipo de manera regular. }\end{array}$ & $\begin{array}{l}\text { Transaccional: la focalización está en el adecuado inter- } \\
\text { cambio de recursos. Implica que el líder define las tareas } \\
\text { al subordinado y a cambio de su desempeño el trabajador } \\
\text { se hace acreedor a una recompensa. }\end{array}$ \\
\hline
\end{tabular}

la percepción de los líderes entrevistados coincide en que el liderazgo es una habilidad gerencial que se utiliza para elevar la productividad empresarial, lo que justifica la pertinencia de seguir avanzando desde la comunidad académica científica en el estado del arte de este fenómeno. Para delimitar el alcance geográfico de la investigación, cobra importancia enfocar el estudio de liderazgo gerencial en el estado de Tamaulipas, México, porque es la entidad que tiene la tasa de desempleo más alta de este país $(7,12 \%)$ y de acuerdo con Robles (2011), forma parte de los diez principales Estados caracterizados por la mortandad de sus empresas durante el 2010.

\section{Metodología}

\subsection{Diseño de la investigación y caracteriza- ción del estudio}

El proceso de planificación seguido para la ejecución de esta investigación, a fin de dar respuesta a la pregunta general establecida que refiere a ¿Cuáles son las prácticas de liderazgo que ejercen los gerentes comerciales de pequeñas y medianas empresas localizadas en Tamaulipas, México? y dar cumplimiento a los objetivos general y específicos de indagación (que se detallan en el apartado de introducción de este trabajo), se esquematiza de manera general en la Ilustración 1.

El alcance de este estudio fue descriptivo, debido al interés de identificar y caracterizar los comportamientos de la gerencia comercial en las cinco prácticas de liderazgo del modelo IPL de Kouzes \& Posner $(1997,2003)$. Se trató de un diseño de investigación no experimental, ya que no se influyó en los encuestados o en su entorno para que respondieran a las preguntas formuladas en el cuestionario. Se llevó a cabo un estudio transversal, con motivo de que la recolección de datos ocurrió en un periodo específico del tiempo, actividad de campo que se realizó de noviembre a diciembre de 2013.

La investigación que se realizó corresponde al enfoque cuantitativo, puesto que se recabaron los datos por medio de cuestionario y se analizaron los mismos con técnicas como el Análisis Factorial Exploratorio (AFE), para determinar la estructura subyacente de las dimensiones o prácticas de liderazgo; el Alpha de Cronbach para la valoración de la confiabilidad del instrumento, se corrieron prueba t y ANOVA de un factor para el contraste de medias (Mertens, 2010;

1 Fuente: Elaboración a partir de Kouzes \& Posner (2003); Robles (2008); Robles, De la Garza \& Medina (2008); Cuadra \& Veloso (2009); Zarate \& Matviuk (2012). 
Ilustración 1. Diseño metodológico de la investigación²

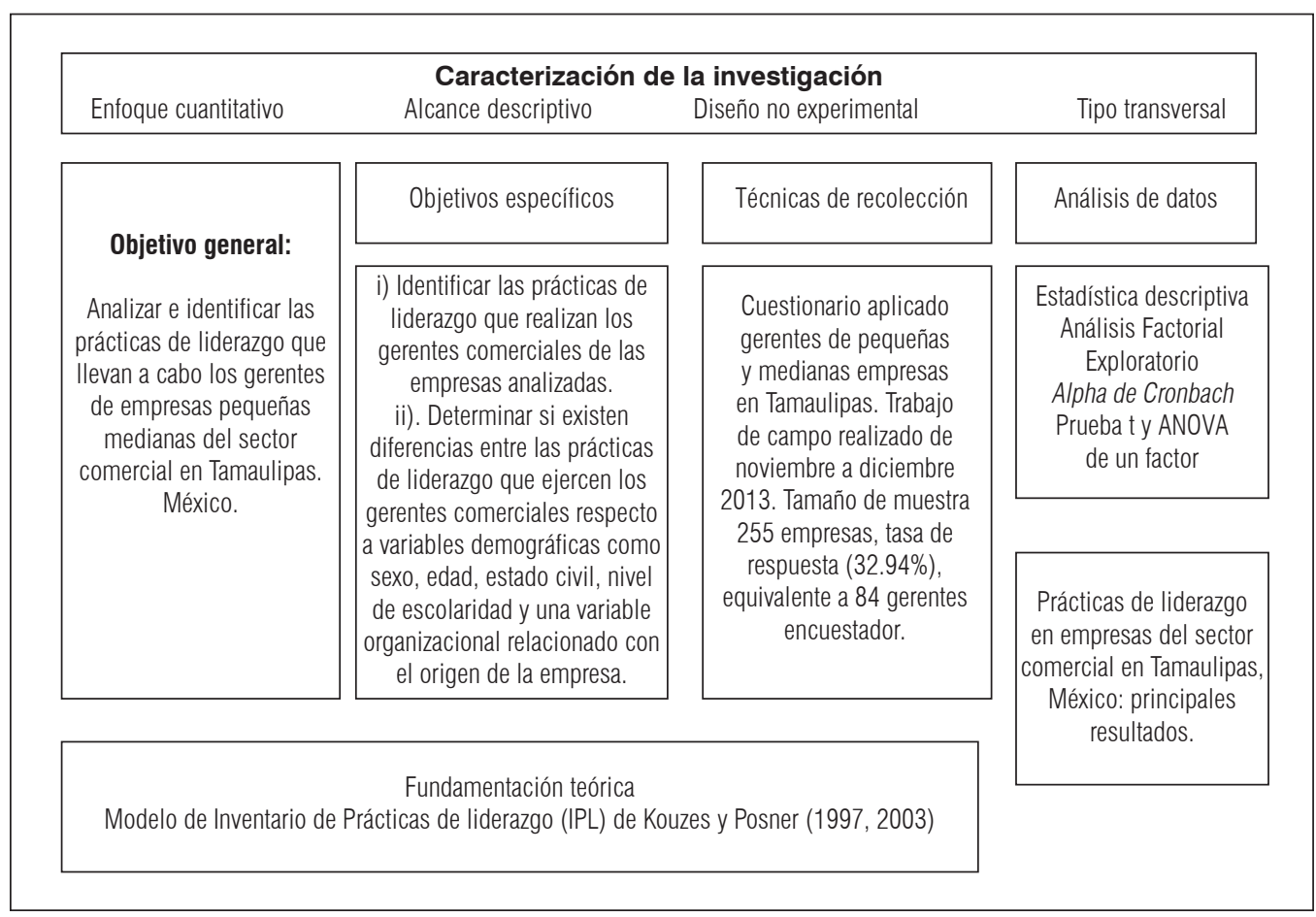

Hernández, Fernández \& Baptista, 2010; Creswell, 2012).

\subsection{Población de estudio y tamaño de la muestra}

Para esta investigación los sujetos clave de la información son los gerentes de las empresas pequeñas y medianas del sector comercial en Tamaulipas, México (Tabla 2). El criterio para categorizar a las organizaciones de acuerdo a su tamaño, fue el número de empleados. Las pequeñas ocupan de 11 a 50 personas y las medianas de 51 a 250 . Este criterio de estratificación fue establecido en México por la Secretaría de Economía en el año 2002. Se optó por aplicar éste, a pesar de la fecha, porque es difícil el acceso a datos relacionados con el total de ventas netas anuales, los ingresos y activos fijos, puesto que la gerencia es muy sensible en proporcionar esta información en la región, por las condiciones de inseguridad que prevalece en la entidad.
Para efecto de determinar el número de empresas que podían ser consideradas parte de este estudio, se decidió observar las estadísticas presentadas en el Sistema de Información Empresarial Mexicano (SIEM). Se decidió por la información de este siste$\mathrm{ma}$, a diferencia de otras bases de datos, porque las empresas que se incorporan al SIEM, lo hacen de manera voluntaria, ello permite estimar que era posible lograr un nivel de participación aceptable en esta investigación por la gerencia. En base al rango de empleados, se identificó que existen en Tamaulipas 16.436 unidades económicas en el sector comercial, distribuidas conforme se describe en la Tabla 2.

En base a la información que se consultó en el SIEM, se determinó que del total de empresas comerciales en Tamaulipas (16.436), sólo 760 unidades económicas correspondían a la categoría de pequeñas y medianas organizaciones. Por lo que este dato representó la población de empresas a considerar en la presente investigación. Para la determinación del

\footnotetext{
2 Fuente: Elaboración autores.
} 
tamaño de muestra, se aplicó la fórmula de muestras finitas, tomando como base un nivel de confianza del $95 \%$ y un error del $5 \%$.

$$
\mathrm{n}=\frac{\mathrm{Z}^{2} \cdot \mathrm{N} \cdot \mathrm{p} \cdot \mathrm{q}}{\mathrm{i}^{2}(\mathrm{~N}-1)+\mathrm{Z}^{2} \cdot \mathrm{p} \cdot \mathrm{q}}
$$

Donde:

$\mathrm{n}=$ tamaño de la muestra;

$\mathrm{N}=$ tamaño de la población;

$\mathrm{Z}=$ valor de la distribución normal;

$\mathrm{p}=$ prevalencia esperada del parámetro a evaluar;

$\mathrm{q}=(1-\mathrm{p})$

$\mathrm{i}=$ error esperado, por lo que el tamaño de la muestra resulta como sigue:

$$
\mathrm{n}=\frac{1,96^{2} \cdot 760 \cdot 0,5 \cdot 0,5}{0,05^{2}(760-1)+1,96^{2} \cdot 0,5 \cdot 0,5}=255 \text { empresas }
$$

En la inmersión en el trabajo de campo, previo a la invitación oficial a los gerentes para participar en el estudio, por las condiciones de inseguridad que se viven en el estado de Tamaulipas, y con el propósito fundamental de lograr confianza y participación de la gerencia en esta investigación, se estableció comunicación en el mes de octubre 2013, con la Cámara Nacional de Comercio (CANACO), sede Victoria, Tamaulipas, a efecto de establecer por medio de este organismo contacto con las empresas afiliadas a esta corporación y a otras sedes. Producto del respaldo de esta asociación, sólo se logró contar con la participación voluntaria de 84 gerentes que accedieron participar en el estudio. En base a lo anterior, se señala que se constituyó una muestra no probabilis- ta y a conveniencia para este estudio, aplicándose cuestionario en los meses de noviembre y diciembre de 2013, únicamente a los directivos que voluntariamente aceptaron de las 255 empresas que se les envío invitación para formar parte de la investigación (equivalente a una tasa de respuesta del $32,94 \%$ ).

\subsection{Técnica de recolección de datos, evaluación de su confiabilidad y validez}

En cuanto al diseño del cuestionario aplicado en este estudio, se señala que con el fin de cuidar la validez de contenido del constructo, se tomó como referente la escala desarrollada en el Modelo de IPL de Kouzes \& Posner $(1997,2003)$. Esta decisión se fundamentó en Bass \& Riggio (2006), quienes señalan que el instrumento para medir el comportamiento de líderes transformacionales y para desarrollar programas de liderazgo precisamente es el IPL. Por lo que se adaptó la redacción de los ítems al contexto empresarial de análisis. Una vez realizada la adaptación, se solicitó a expertos en el área administración y desarrollo organizacional la revisión del instrumento. Esta evaluación con expertos dio lugar a modificaciones, principalmente en la redacción de los enunciados, ambigüedad de términos y redundancia en los ítems. Posteriormente, se llevó a cabo una muestra piloto del cuestionario, encuestando a 20 empresarios, dando como resultado el cambio de redacción en los ítems en el lenguaje empresarial que manejan.

Finalmente, el cuestionario quedó conformado por dos secciones. La primera contempló datos del encuestado (sexo, edad, escolaridad, estado civil) y una

Tabla 2. Empresas comerciales en Tamaulipas, México: su clasificación ${ }^{3}$

\begin{tabular}{|c|c|c|c|}
\hline $\begin{array}{c}\text { Tamaño de la } \\
\text { empresa }\end{array}$ & $\begin{array}{c}\text { Rango de } \\
\text { empleados }\end{array}$ & $\begin{array}{c}\text { Número de unidades } \\
\text { económicas por tamaño } \\
\text { de empresa }\end{array}$ & $\begin{array}{c}\text { Total de empresas } \\
\text { comerciales en } \\
\text { Tamaulipas, México }\end{array}$ \\
\cline { 1 - 3 } Micro & $0-10$ & 15.669 & \multirow{2}{*}{16.436 unidades económicas } \\
\cline { 1 - 3 } Pequeña & $11-50$ & 664 & \\
\hline Mediana & $51-250$ & 96 & \\
\hline Grande & 251 a mas & 7 & \\
\hline
\end{tabular}

3 Fuente: SIEM (2013). 
pregunta relacionada con el origen de la empresa (propia, familiar, varios socios). La segunda sección se enfocó a la recolección de datos sobre las cinco prácticas de liderazgo (Tabla 3), que correspondía a la percepción que el directivo tiene sobre la frecuencia de su práctica ante el comportamiento descrito. Esta segunda sección, contenía 30 ítems divididos en seis preguntas para cada una de las cinco dimensiones (Tabla 3) que propone el modelo de IPL de Kouzes \& Posner (1997, 2003). Las preguntas se presentaron al gerente encuestado en escala tipo Likert de cinco puntos ( $1=$ Raramente o Nunca, $2=$ De vez en cuando, 3= Ocasionalmente, $4=\mathrm{A}$ menudo, y $5=$ Muy a menudo o Siempre).

Para analizar la confiabilidad del cuestionario en esta investigación, se aplicó Alpha de Cronbach, este indicador establece que un instrumento tiene consistencia interna cuando se determina un valor de 0,70 como mínimo (Nunnally, 1978). En este sentido, la escala global obtuvo 0,95 , además por cada práctica o dimensión del IPL mostró valores superiores al mínimo establecido (Tabla 3), infiriendo en este estudio un nivel aceptable y alto de confiabilidad del instrumento.

\subsection{Técnicas de análisis de datos}

Para identificar la estructura subyacente de prácticas de liderazgo empresarial en el sector comercial en Tamaulipas, México, se ejecutó la técnica Análisis Factorial Exploratorio (AFE), por el método de componentes principales y rotación Varimax (Hair, Anderson, Tatham \& Black, 2008). Para aplicar esta técnica se utilizó la versión 18 del paquete estadístico SPSS (Statistical Package for the Social Sciences). Con este análisis se confirmó en el presente estudio, las cinco dimensiones (Tabla 3) del modelo de IPL desarrollado por Kouzes \& Posner (1997, 2003), puesto que se identificaron cinco dimensiones o factores (con eigenvalues mayores que 1), con valores dentro de los parámetros recomendados para el KMO (superior a 0,70 ) y las correlaciones cercanas a 1,00 (Segars, 1997; Sledgianowski, Luftman \& Reilly, 2006). Así, el KMO de cada práctica de liderazgo se encuentra entre 0,74 y 0,78 ; y la correlación total presentó indicadores significativos. Lo que corrobora la estructura de cinco dimensiones, en concordancia con el modelo IPL que fue la base en este estudio.

Tabla 3. Confiabilidad de la escala de medición de prácticas de liderazgo ${ }^{4}$

\begin{tabular}{|c|c|c|c|}
\hline $\begin{array}{l}\text { Dimensión del Modelo } \\
\text { IPL }\end{array}$ & $\begin{array}{c}\text { No. de Ítem } \\
\text { (Detalle en Tabla 4) }\end{array}$ & $\begin{array}{l}\text { Alfa de } \\
\text { Cronbach }\end{array}$ & $\begin{array}{l}\text { Análisis Factorial Exploratorio } \\
\text { (AFE) }\end{array}$ \\
\hline $\begin{array}{l}\text { 1. Desafiar los procesos } \\
\text { (DP) }\end{array}$ & $1,6,11,16,21,26$ & 0,78 & $\begin{array}{c}\text { KM0 0,767 } \\
\text { Chi2 149,828 } \\
\text { Sig. 0,000 }\end{array}$ \\
\hline $\begin{array}{l}\text { 2. Inspirar una visión compartida } \\
\text { (IVC) }\end{array}$ & $2,7,12,17,22,27$ & 0,82 & $\begin{array}{c}\text { KM0 0,741 } \\
\text { Chi2 210,311 } \\
\text { Sig. 0,000 }\end{array}$ \\
\hline $\begin{array}{l}\text { 3. Habilitar a los demás para que actúen } \\
\text { (HDA) }\end{array}$ & $3,8,13,18,23,28$ & 0,81 & $\begin{array}{c}\text { KM0 0,787 } \\
\text { Chi2 168,360 } \\
\text { Sig. 0,000 }\end{array}$ \\
\hline $\begin{array}{l}\text { 4. Modelar el camino } \\
\text { (MC) }\end{array}$ & $4,9,14,19,24,29$ & 0,83 & $\begin{array}{c}\text { KMO 0,785 } \\
\text { Chi2 195,265 } \\
\text { Sig. 0,000 }\end{array}$ \\
\hline $\begin{array}{l}\text { 5. Dar aliento al corazón } \\
\text { (DC) }\end{array}$ & $5,10,15,20,25,30$ & 0,82 & $\begin{array}{c}\text { KM0 0,763 } \\
\text { Chi2 194,957 } \\
\text { Sig. 0,000 }\end{array}$ \\
\hline \multicolumn{4}{|c|}{ Escala completa Alfa de Cronbach 0,95 } \\
\hline
\end{tabular}

4 Fuente: Elaboración autores. 
En relación con los datos recopilados sobre edad, sexo, escolaridad, estado civil y origen de la empresa, se aplicó estadística descriptiva (frecuencias y porcentaje), en primer instancia para categorizar a los sujetos encuestados y posteriormente para efecto de determinar las medias por cada práctica de liderazgo determinada: i) desafiar los procesos, ii) inspirar una visión compartida, iii) habilitar a los demás para actuar, iv) modelar el camino y, v) dar aliento al corazón. Posteriormente, para realizar el análisis de las variables de datos generales de los encuestados y de origen de la empresa, a fin de determinar si existen diferencias significativas con las prácticas de liderazgo, se aplicaron las técnicas de prueba t de Student y ANOVA de un factor.

\section{Resultados}

En relación con el perfil de los gerentes encuestados, se determinó en esta investigación que un porcentaje mayoritario son hombres (67\%) y únicamente el $33 \%$ mujeres. Esta tendencia en la baja participación de la mujer en cargos gerenciales en las organizaciones, coincide con estudios previos, en donde se ha determinado esta desigualdad (Estebaranz \& Mingorance, 2005; Ramos, 2005; Cáceres, Trujillo, Hinojo, Aznar \& García, 2012; Contreras, Pedraza \& Mejía, 2012).

En relación con el estado civil, un porcentaje alto de los gerentes está casado $(65,5 \%)$, el $28,6 \%$ son solteros y una minoría están divorciados $(2,4 \%)$ o viudos (1,2\%). Respecto al nivel de escolaridad de los gerentes analizados, se identificó que el 58,3\% tiene estudios de licenciatura y un $8,3 \%$ cuenta con posgrado, el 20,2\% tiene educación media (bachillerato), un $4,8 \%$ estudios comerciales y el $8,3 \%$ tiene el nivel de secundaria. Estos resultados en estas tres variables (sexo, estado civil y nivel de escolaridad) son muy cercanos a los generados por Robles, De la Garza \& Medina (2008) en un estudio anterior en la región.

El 27,5\% tiene de 34 a 41 años, el 20\% oscilan en un rango de edad de 42 a 49 años, para los rangos de edad de 26 a 33 años se sitúan con un $18.8 \%$, mientras que un $16,3 \%$ de 18 a 25 años, el resto de los encuestados (8,8\%) tienen de 50 años en adelante. El $17,9 \%$ de los gerentes informa que la empresa es propia, el $39,3 \%$ es familiar y un alto porcentaje señala que la empresas es propiedad de varios socios (42,9\%). El $61 \%$ de los encuestados se localizó en un nivel de gerente funcional, el $21 \%$ como gerente general y el $16 \%$ como el propietario del negocio.

En relación con el objetivo general de investigación trazado en el presente trabajo, que refiere al análisis e identificación de las prácticas de liderazgo que llevan a cabo los gerentes de las empresas comerciales, se encontró que las medias de las cinco prácticas determinadas, muestran un comportamiento muy similar, que permite deducir que los gerentes comerciales en Tamaulipas, México, expresan que a menudo llevan a cabo los comportamientos o conductas compren-

Ilustración 2. Medias generales de IPL en gerencia de empresas comerciales en Tamaulipas, México ${ }^{5}$

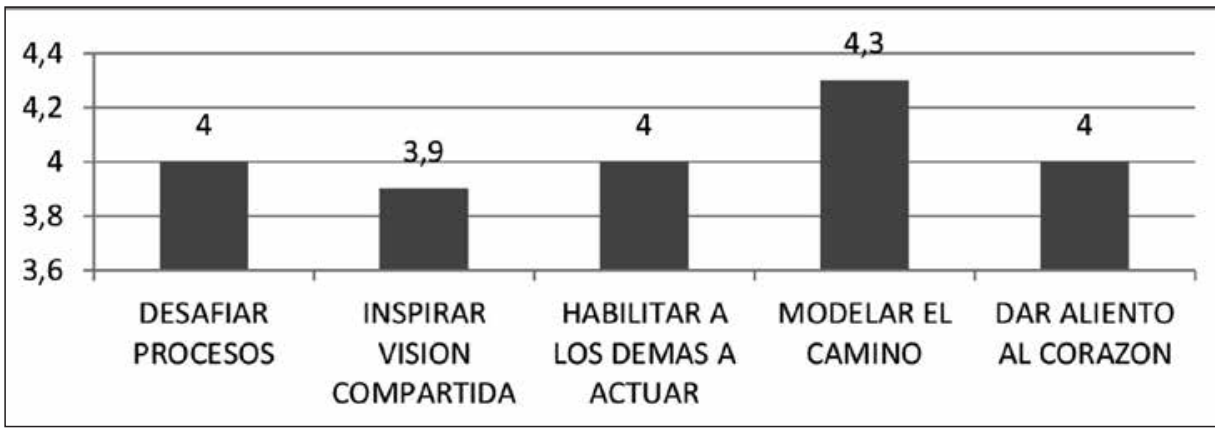

5 Fuente: Elaboración autores. 
didas en las dimensiones de desafiar los procesos, inspirar una visión compartida, habilitar a los demás para actuar, modelar el camino y dar aliento al corazón (Ilustración 2). Se infiere por tanto, que las prácticas de liderazgo de los gerentes comerciales de las empresas analizadas, corresponden a los comportamientos de las cinco dimensiones del modelo de IPL, este resultado coincide con estudios previos realizados (Robles, De la Garza \& Medina, 2008).

Cabe señalar que para la interpretación de la intensidad en que las prácticas de liderazgo son realizadas por los gerentes de las empresas comerciales estudiadas, se estableció el criterio de tres niveles: i) incipiente, que agrupó medias de 0 a 3,5; ii) moderado, conformado por medias de 3,6 a 4,5 y el tercero, iii) nivel óptimo corresponde a medias de 4,6 en adelante. En el marco de este criterio de análisis, se observa en términos generales (Ilustración 2), que las cinco prácticas de liderazgo se caracterizan en un nivel moderado en la gerencia comercial de las empresas analizadas en Tamaulipas.

Se ha observado que a pesar de que la media general de las cinco prácticas de liderazgo de los gerentes de las empresas comerciales estudiadas, muestra un nivel moderado (medias de 3,6 a 4,5, Ilustración 2), hallazgo que se estima aceptable, existen porcentajes importantes de gerentes que es necesario desarrollen sus habilidades en los comportamientos específicos de las dimensiones del modelo de IPL, como se expone en las inferencias descritas a continuación. De hecho, el comportamiento con la media más baja, refiere a la constancia en la práctica de los valores con que se compromete la gerencia (Tabla 4).

De hecho, al observar las conductas o comportamientos específicos de los líderes en cada dimensión del modelo IPL, se identificó que una de las conductas que se practica menos refiere a dedicar tiempo a celebrar el logro de objetivos (Tabla 4), que corresponde a la dimensión de dar aliento al corazón, puesto que un $47,7 \%$ manifiesta que nunca o bien ocasionalmente celebra la consecución de objetivos en la organización, hallazgo coincidente con el deter- minado por Robles, De la Garza \& Medina (2008). De hecho en esta misma dimensión se identificó otro comportamiento que refuerza la debilidad anterior, ya que el $39,3 \%$ expresó que de nunca a ocasionalmente encuentra maneras de celebrar los logros.

Tabla 4. Medias de ítems que formaron parte del constructo IPL 6

\begin{tabular}{|c|c|}
\hline $\begin{array}{l}\text { 1. Busco oportunidades de reto que pongan a prueba mis apti- } \\
\text { tudes. }\end{array}$ & 3,9 \\
\hline $\begin{array}{l}\text { 2. Describo a los demás el futuro que me gustaría que labráramos } \\
\text { juntos. }\end{array}$ & 3,9 \\
\hline $\begin{array}{l}\text { 3. Involucro a los demás en la planeación de las acciones que } \\
\text { emprenderemos. }\end{array}$ & 4 \\
\hline 4. Tengo muy clara mí propia filosofía acerca del liderazgo. & 4,3 \\
\hline $\begin{array}{l}\text { 3.75. Dedico tiempo a celebrar el logro de cada objetivo interme- } \\
\text { dio del proyecto. }\end{array}$ & 3,5 \\
\hline $\begin{array}{l}\text { 6. Cuestiono nuestra manera de hacer las cosas en la organización } \\
\text { donde trabajo. }\end{array}$ & 3,7 \\
\hline $\begin{array}{l}\text { 7. Invito a los demás a que compartan mis sueños del futuro como } \\
\text { si fueran los suyos propios. }\end{array}$ & 3,7 \\
\hline $\begin{array}{l}\text { 8. Concedo a los demás libertad suficiente para que tomen sus } \\
\text { propias decisiones. }\end{array}$ & 3,8 \\
\hline $\begin{array}{l}\text { 9. Soy constante en la práctica de los valores con los que me } \\
\text { comprometo. }\end{array}$ & 3,3 \\
\hline 10. Alabo a quienes realizan un buen trabajo. & 4,2 \\
\hline $\begin{array}{l}\text { 11. Busco maneras innovadoras de mejorar lo que hacemos en } \\
\text { nuestra organización. }\end{array}$ & 4,1 \\
\hline $\begin{array}{l}\text { 12. Comunico con claridad una perspectiva positiva y llena de es- } \\
\text { peranzas para el futuro de nuestra organización. }\end{array}$ & 4 \\
\hline $\begin{array}{l}\text { 13. Genero una atmósfera de confianza mutua en los proyectos } \\
\text { que dirijo. }\end{array}$ & 4,2 \\
\hline $\begin{array}{l}\text { 14. Me aseguro de que el equipo de trabajo plantee metas claras, } \\
\text { haga planes y fije objetivos intermedios para cada proyecto que } \\
\text { dirijo. }\end{array}$ & 4,1 \\
\hline 15. Encuentro maneras de celebrar los logros. & 3,7 \\
\hline $\begin{array}{l}\text { 16. Experimento y me arriesgo con nuevos métodos de trabajo, } \\
\text { incluso a sabiendas de que me expongo al fracaso. }\end{array}$ & 3,8 \\
\hline $\begin{array}{l}\text { 17. Señalo a los demás cómo pueden materializar sus propios in- } \\
\text { tereses a largo plazo si todos participan de una visión en común. }\end{array}$ & 3,8 \\
\hline $\begin{array}{l}\text { 18. Logro que los demás sientan suyos los proyectos en que tra- } \\
\text { bajan. }\end{array}$ & 4 \\
\hline
\end{tabular}

6 Fuente: Elaboración autores. 


\begin{tabular}{|l|l|}
\hline $\begin{array}{l}\text { 19. Me muestro congruente con mí forma de actuar y lo que pienso } \\
\text { acerca de lo que debe ser el trabajo. }\end{array}$ & 4,3 \\
\hline $\begin{array}{l}\text { 20. Señalo intencionalmente al resto de la organización el buen } \\
\text { trabajo realizado por mí equipo. }\end{array}$ & 4 \\
\hline $\begin{array}{l}\text { 21. Experimento, corro riesgos y aprendo de los errores que se } \\
\text { producen en mí trabajo. }\end{array}$ & 4,1 \\
\hline $\begin{array}{l}\text { 22. Miro hacia adelante y predigo como espero que sea nuestro } \\
\text { futuro. }\end{array}$ & 4,2 \\
\hline $\begin{array}{l}\text { 23. Me preocupo por el nivel de capacitación que mis colaborado- } \\
\text { res deben tener en su trabajo. }\end{array}$ & 4,2 \\
\hline $\begin{array}{l}\text { 24. Soy congruente en cuanto a mi forma de actuar y pensar con } \\
\text { respecto a mí trabajo. }\end{array}$ & 4,4 \\
\hline 25. Reconozco y aliento la creatividad de mis colaboradores. & 4,3 \\
\hline $\begin{array}{l}\text { 26. Promuevo la mejora continua y la innovación en los procedi- } \\
\text { mientos de trabajo. }\end{array}$ & 4,2 \\
\hline $\begin{array}{l}\text { 27. Contagio a los demás mi emoción y entusiasmo en cuanto a } \\
\text { las posibilidades futuras. }\end{array}$ & 4,2 \\
\hline $\begin{array}{l}\text { 28. Habilito en mí grupo de trabajo la participación y la toma de } \\
\text { decisiones. }\end{array}$ & 4 \\
\hline $\begin{array}{l}\text { 29. Predico con el ejemplo en lo referente al compromiso insti- } \\
\text { tucional. }\end{array}$ & 4,3 \\
\hline $\begin{array}{l}\text { 30. Reconozco las contribuciones individuales de mis colaborado- } \\
\text { res en el éxito de cualquier proyecto. }\end{array}$ & 4,2 \\
\hline
\end{tabular}

Al estudiar el resto de los comportamientos del líder, en la dimensión de desafiar los procesos se identificó en un nivel incipiente un porcentaje importante $(34,5 \%)$ de los gerentes que de nunca a ocasionalmente cuestionan la manera de hacer las cosas en la organización donde trabajan y el 34,5\% señaló que nunca o bien ocasionalmente experimenta y se arriesga con nuevas maneras de hacer el trabajo. Esto revela que un porcentaje alto de los gerentes no está preparado para hacer frente al cambio y a la innovación, variables que son una constante en el entorno competitivo actual. En la dimensión de habilitar a los demás para que actúen, se encontró que el $35,7 \%$ manifiesta que nunca o bien ocasionalmente conceden libertad a sus subalternos para que tomen sus propias decisiones.

Respecto a la dimensión de inspirar una visión compartida (con media más baja), se encontraron porcentajes importantes de gerentes que nunca o bien ocasionalmente (nivel incipiente) llevan a cabo algunos compartimientos o conductas que caracterizan a estas prácticas de liderazgo, por ejemplo: el 35,7\% mani- fiesta no señalar con frecuencia a los demás como pueden materializar sus propios intereses a largo plazo si todos comparten una visión en común, el 33,3\% no invita a los demás a compartir sus sueños de futuro, y el $31 \%$ manifestó que de nunca a ocasionalmente comunica con claridad una perspectiva positiva y llena de esperanzas para el futuro de la organización.

En relación con el objetivo específico de investigación, que consistía en determinar si existían diferencias en las prácticas de liderazgo de los gerentes comerciales, sobre variables como el sexo, edad, estado civil, escolaridad y el origen de la empresa, se encontraron los siguientes resultados. Se identificó respecto al sexo, que tanto hombres como mujeres valoran de la misma manera las cinco prácticas de liderazgo: desafiar los procesos, inspirar una visión compartida, habilitar a los demás para actuar, modelar el camino y dar aliento al corazón, no encontrándose diferencias significativas entre ellos (prueba $\mathrm{t}$, con valores $\mathrm{Pv}>$ a 0,05 ).

Al estudiar la relación de la variable origen de la empresa (propia, familiar, de varios socios) con las prácticas de liderazgo del modelo IPL, se identificó que existen diferencias significativas, al determinarse que los gerentes de empresas que son propiedad de varios socios valoran más alto las dimensiones de habilitar a los demás para actuar $(\mathrm{F}=4,574, \mathrm{Pv}=0,01)$ y la de modelar el camino $(\mathrm{F}=3,941, \mathrm{Pv}=0,02)$, que aquellos quienes se encuentran en empresas de carácter propio o familiar (Tabla 5).

Al correr la prueba ANOVA de un factor para la variable de rango de edad, estado civil y nivel de escolaridad, no se determinaron diferencias estadísticamente significativas en relación con las cinco prácticas de liderazgo del Modelo IPL, tanto los gerentes jóvenes como los de mayor edad valoran con la misma importancia los comportamientos en las cinco dimensiones: desafiar los procesos, inspirar una visión compartida, habilitar a los demás para actuar, modelar el camino y dar aliento al corazón, así mismo no se encontraron diferencias significativas respecto a las variables categóricas de estado civil y nivel de escolaridad respecto a las dimensiones del IPL ( F con valores $\mathrm{Pv}>$ a 0,05$)$. Con los resultados anteriores se ha mostrado la caracterización de la relación entre 
Tabla 5. Análisis de contraste de medias, variable de origen de la empresa y las prácticas de liderazgo del modelo IPL

\begin{tabular}{|c|c|c|c|c|c|c|c|}
\hline $\begin{array}{c}\text { Origen de la } \\
\text { empresa }\end{array}$ & $\mathbf{N}$ & Estadísticos & DP & IVC & HDA & MC & DC \\
\hline \multirow{2}{*}{ Propia } & \multirow{2}{*}{15} & Media & 3,84 & 3,81 & 3,74 & 3,97 & 3,76 \\
\cline { 3 - 9 } & & Desviación típica & 0,673 & 0,59 & 0,793 & 0,806 & 0,725 \\
\hline \multirow{2}{*}{ Familiar } & \multirow{2}{*}{33} & Media & 3,84 & 3,92 & 3,94 & 4,33 & 3,93 \\
\cline { 3 - 9 } & & Desviación típica & 0,673 & 0,763 & 0,664 & 0,555 & 0,756 \\
\hline \multirow{2}{*}{ De varios socios } & \multirow{2}{*}{36} & Media & 4,21 & 4,12 & 4,3 & 4,49 & 4,22 \\
\cline { 3 - 9 } & & Desviación típica & 0,713 & 0,695 & 0,61 & 0,539 & 0,67 \\
\hline \multirow{2}{*}{ Total } & \multirow{2}{*}{84} & Media & 4 & 3,99 & 4 & 4,34 & 4,03 \\
\cline { 3 - 9 } & & Desviación típica & 0,707 & 0,708 & 0,694 & 0,62 & 0,729 \\
\cline { 3 - 9 } & & F & 3,01 & 1,259 & 4,574 & 3,941 & 2,645 \\
\hline
\end{tabular}

variables generales de los gerentes encuestados con respecto a las cinco dimensiones del IPL.

\section{Conclusiones e implicaciones para futuras investigaciones}

El liderazgo ha sido un tema ampliamente estudiado, pero enfocado normalmente a grandes empresas públicas y privadas, siendo importante continuar realizando investigaciones que cada vez más incorporen el análisis de las prácticas de liderazgo de los gerentes de las pequeñas y medianas organizaciones, por la necesidad que existe de contribuir a elevar la competitividad y productividad de estas entidades económicas, ya que son factores clave en la economía de las naciones, y esencialmente el liderazgo que sus gerentes ejercen podría llegar a ser el elemento que haga la diferencia para generar ventajas competitivas que les permita su longevidad.

Se observó en este estudio que las cinco dimensiones del IPL (desafiar los procesos, inspirar una visión compartida, habilitar a los demás para actuar, modelar el camino y dar aliento al corazón), están presentes en las prácticas de liderazgo de la gerencia comercial de las empresas analizadas, en Tamaulipas, México. Sin embargo, se señala que se identificó que el origen de la empresa es una variable que mostró diferencias significativas en dos de las cinco dimen- siones del modelo IPL: i) habilitar a los demás para actuar y ii) en modelar el camino, encontrando que los gerentes de las organizaciones que son propiedad de varios socios valoran más alto los comportamientos del líder en estas dos dimensiones de quienes están en empresas familiares o que son propietarios. De allí que en futuros estudios, sería relevante llevar a cabo entrevistas a profundidad para identificar los factores que inciden en estos hallazgos.

En los comportamientos específicos de los líderes en cada dimensión del modelo IPL, se identificó que dentro de las conductas que se practican menos en porcentajes importantes de los gerentes comerciales estudiados, son las referentes a: i) dedicar tiempo a celebrar el logro de objetivos, ii) encontrar maneras de celebrar los logros, iii) en buscar nuevas formas de hacer las cosas en la organización donde trabajan, iv) en experimentar y arriesgarse con nuevas maneras de hacer el trabajo, v) en conceder libertad a sus subalternos para que tomen sus propias decisiones, vi) no señalar con frecuencia a los demás como pueden materializar sus propios intereses a largo plazo si todos comparten una visión en común, vii) en no invitar a los demás a compartir sus sueños de futuro, viii) en comunicar con claridad una perspectiva positiva y llena de esperanzas para el futuro de la organización. Estos hallazgos a pesar de que la mayoría de los gerentes tiene niveles altos de

7 Fuente: Elaboración autores. 
estudios (licenciatura y posgrado), revelan en gran parte que los gerentes comerciales de esta región no están preparados para hacer frente al cambio y a la innovación, variables que son una constante en el entorno competitivo actual.

En la relación de variables de datos generales de los gerentes comerciales encuestados (sexo, edad, estado civil, escolaridad) con las prácticas de liderazgo del modelo IPL, no se encontraron diferencias significativas, tanto hombres como mujeres valoran de la misma manera las cinco prácticas de liderazgo (desafiar los procesos, inspirar una visión compartida, habilitar a los demás para actuar, modelar el camino y dar aliento al corazón); así mismo la edad, el estado civil y la escolaridad no se encontró diferencias significativas entre los grupos de gerentes comerciales participantes en esta investigación.

Se encontró en esta investigación, que la presencia del hombre es mayoritario en relación con la mujer en los puestos gerenciales de las empresas analizadas en Tamaulipas. Sin lugar a dudas, este hallazgo coincide con estudios previos sobre el tema en cuestión, y representa una área de interés para que en investigaciones posteriores en esta región, se profundice en el análisis de la relación del género y el liderazgo, a fin de identificar los factores que inciden en esta desigualdad y proponer estrategias que lleven a transitar a una mayor equidad en la participación de la mujer en el nivel gerencial de las organizaciones. Sobre todo porque el estilo de dirección que ejercen las mujeres, tiende a ser más conveniente en el entorno que enfrentan las empresas hoy en día. Sin embargo, resulta necesario contribuir a que la mujer ejerza el liderazgo desde sus propias cualidades, en vez de imitar el rol tradicional del líder masculino, siendo relevante llevar a cabo estudios de variables psicológicas, sociales y culturales con abordaje de diferentes métodos de análisis, a fin de alcanzar una mayor y mejor compresión de este fenómeno de desigualdad (Contreras, Pedraza \& Mejía, 2012).

El liderazgo es un fenómeno que no solo depende directamente de las personas que lo ejercen, también está influenciado por el medio en que se desenvuelva y de la organización en que se desempeña. De allí que en futuras investigaciones es pertinente estudiar la relación del liderazgo con variables como el clima organizacional, la satisfacción laboral, el compromiso organizacional, el trabajo en equipo, la cultura organizacional, la ubicación dentro de la estructura organizacional, la eficacia y desempeño de las empresas, las cualidades y rasgos del líder, los estilos de liderazgo, solo por mencionar algunos factores que podrían potenciar la efectividad del liderazgo que ejercen los gerentes al frente de las pequeñas y medianas empresas.

Dentro de las limitaciones de este estudio, se señala que los resultados no pueden tomarse como generalizables a todas las empresas comerciales de Tamaulipas, México, debido al bajo número de gerentes que accedieron voluntariamente participar (muestra voluntaria de 84 directivos), por lo que en futuros análisis será necesario determinar muestras representativas de la población de análisis y establecer estrategias de contacto para lograr una mayor participación de organizaciones de este sector y de otros giros.

\section{Referencias}

Amorós, E. (2007). Comportamiento organizacional: en busca del desarrollo de ventajas competitivas. Lambayeque, Perú: USAT Escuela de economía.

Arredondo, F. (2011). La integridad del líder de empresa. México: Ediciones Eón.

Aronson, E. (2001). Integrating leadership styles and ethical perspectives. En: Canadian Journal of Administrative Sciences, 18(4): 244-256.

Arroyo, I. (2005). La cultura y el proceso de globalización en el desarrollo de las pymes. En: Revista Universisad de Costa Rica: 1-15.

Barahona, H., Cabrera, D. \& Torres, U. (2011). Los líderes en el siglo XXI. En: Entramado, 7(2): 86-97.

Bass, B. (1985). Leadership and performance beyond expectations. New York: The Free Press.

Bass, B. (1990). From transactional to transformational leadership: learning to share the vision. En: Organizational Dynamics: 19-31.

Bass, B. (1998). The ethics of transformational leadership. En: Ciulla, J. (ed.) Ethics the heart of leadership. Wesport, CT: Praeger.

Bass, B. (1999). Two decades of research and development in transformational leadership. En: European Journal of Work and Organizational Psycology, 8(1): 9-32.

Bass, B. \& Riggio, R. (2006). Transformational leadership. Mahwah, USA: Lawrence Erlbaum Associates, Inc.

Boerner, S., Eisenbeiss, S. \& Griesser, D. (2007). Follower behavior and organizational performance: the impact of transformational leaders. En: Journal of Leadership \& Organizational Studies, 13: $15-26$.

Burns, J. (1978). Leadership. USA: Harper and Row.

Cáceres, M., Trujillo, J., Hinojo, F., Aznar, I. \& García, M. (2012). Tendencias actuales de género y el liderazgo de la dirección en los diferentes niveles educativos. En: Educar, 48(1): 69-89. 
CONDUSEF (2013). México. Los errores más comunes del empresario PyME. Recuperado el 19 de septiembre de 2013 de: http:/www.condusef.gob.mx/index.php/empresario-pyme-comousuarios-de-servicios-financieros/548-errores-mas-comunesdel-empresario-pume

Contreras, F. \& Juárez, F. (2013). Efecto del capital psicológico sobre las prácticas de liderazgo en pymes colombianas. En: Revista Venezolana de Gerencia, 18(62): 247-264.

Contreras, F. \& Castro, G. (2013). Liderazgo, poder y movilización organizacional. En: Estudios Gerenciales, 29 (126): 72-76.

Contreras, F., Pedraza, J. \& Mejía, X. (2012). La mujer y el liderazgo empresarial. En: Diversitas: Perspectivas en Psicología, 8 (1): 183-194.

Cortés, A.(2004). Estilos de liderazgo y motivación laboral en el ambiente educativo. En: Revista Ciencias Sociales (Cr), IV(106): 203-214.

Creswell, J. (2012). Educational research: planning, conducting and evaluating quantitative and quialitative research. Boston: Pearson Education Inc.

Cuadra, A. \& Veloso, C. (2009). Grado de supervisión como variable moderadora entre liderazgo y satisfacción, motivación y clima organizacional. En: Ingeniare Revista Chilena de Ingeniería, 18(1): 15-25.

Cuadrado, I., Molero, F. \& Navas, M. (2003). El liderazgo de hombres y mujeres: diferencias en estilos de liderazgo, relaciones entre estilos y predictores de variables de resultado organizacional. En: Acción Psicológica, 2(2): 115-129.

Estebaranz, A. \& Mingorance, P. (2005). Mujeres y liderazgo educativo: una experiencia. El liderazgo pedagógico. Temáticos Escuela. Vol. 14. Madrid: Praxis.

Fiedler, F. (1967). A Theory of Leadership Effectiveness. New York: McGraw-Hill.

Gómez, G., Betancourt, J. \& Zapata, N. (2012). Empresas familiares multigeneracionales. En: Entramado, 8( 2): 3-49.

Guaipatin, C. (2003). Observatorio MiPYME: compilación estadística para 12 países de la región. Informe de Trabajo. Washintong, D.C.: Banco Interamericano de Desarrollo.

Hair, J., Anderson, R., Tatham, R. \& Black, W. (2008). Análisis multivariante. México: Prentice Hall.

Hernández, R., Fernández, C. \& Baptista, P. (2010). Metodología de la investigación. México: McGraw Hill.

INEGI (2010). Resumen de los resultados de los Censos Económicos 2009. Instituto Nacional de Estadística y Geografía - INEGI, México. Recuperado el 8 de septiembre de 2013 de: http:// www.inegi.org.mx/est/contenidos/espanol/proyectos/censos/ ce2009/pdf/RD09-resumen.pdf

Kouzes, J. \& Posner, B. (1997). The leadership challenge. San Francisco. USA: Jossey-Bass INC.

Kouzes, J. \& Posner, B. (2003). Las cinco prácticas de liderazgo ejemplar. San Francisco, Estados Unidos: Pfeiffer editorial John Wiley \& Sons, Inc.

Likert, R. (1961). New patterns of Management. Nueva York: Mcraw-Hill.

Martínez, J. (2006). Factores críticos para el desempeño exportador de las Pymes. En: Tec Empresarial, 1(1): 32-37.

Mendoza, R. \& Ortiz, C. (2006). El liderazgo transformacional, dimensiones e impacto en la cultura organizacional y eficacia de las empresas. En: Revista Facultad de Ciencias Económicas, Universidad Militar Nueva Granada, XIV(1): 118-134.
Mertens, D. (2010). Research and evaluation in education and phsycology: integrating diversity with quantitative, qualitative and mixed methods. Thousand Oaks: Sage Publications.

Molero, F., Recio, P. \& Cuadrado, I. (2010). Liderazgo transformacional y liderazgo transaccional: un análisis de la estructura factorial de Multifactor Leadership Questionnaire (MLQ) en una muestra española. En: Red de Revistas Científicas de América Látina, el Caribe, España y Portugal, 22(3): 495-501.

Nunnally, J. (1978). Psychometric theory. New York,USA: McGraw Hill

Pedraja, L. \& Rodríguez, E. (2004). Efectos del estilo de Liderazgo sobre la eficacia de las organizaciones públicas. En: Revista Facultad de Ingeniería, U.T.A, 12(2): 63-73.

Pedraja, L., Rodríguez, E. \& Rodríguez, J. (2008). Importancia de los estilos de liderazgo sobre la eficacia: un estudio comparativo entre grandes y pequeñas medianas empresas privadas. En: Revista de Ciencias Sociales (RCS), XIV(1): 20-29.

Pedraja, L., Rodríguez, E., Barredo, M., Sagredo, O. \& Segovia, C. (2009). Estilos de liderazgo y resultados del sistema de medicion de la calidad de la educación: un estudio empírico en los colegios básicos de la ciudad de Arica-Chile. En: Revista Chilena de Ingeniería, 17(1): 21-26.

Ramos, M. (2005). Mujeres y liderazgo: una nueva forma de dirigir. Valencia: Universitat de Valencia. Servei de Publicacions.

Robles, S. (2011). Desaparición de empresas en México. En el Sol de Zacatecas. Recuperado el 28 de octubre de 2013 de: http:// www.oem.com.mx/esto/notas/n2061814.htm.

Robles, V. (2008). Una investigación Intra-cultural del liderazgo en México: Igualdad en las PyMES de Hidalgo, Colima y Tamaulipas. En: Revista Facultad de Ciencias Económicas, Universidad Militar Nueva Granada, XVI ( 2): 31-45.

Robles, V., De la Garza, I. \& Medina, J. (2008). El liderazgo de los gerentes de las PyMES de Tamaulipas, México, mediante el Inventario de Prácticas de Liderazgo. En: Cuad. Adm., 21(37): 293-310.

Rodríguez, E. (2010). Estilos de liderazgo, cultura organizativa y eficacia: un estudio empírico en pequeñas y medianas empresas. En: Revista de Ciencias Sociales, XVI (4): 629-641.

Segars, A. (1997). Assessing the unidimensionality of measurement: a paradigma and illustration within the contexto finformation Systems research. En: Omega, 25: 107-121.

Senge, P. (2006). La quinta disciplina: cómo impulsar el aprendizaje en la organización inteligente. Buenos Aires: Granica.

SIEM (2013). Sistema de información empresarial mexicano. Estadísticas por Estado y rango de empleados. Recuperado el 8 de octubre de 2013 de: http://www.siem.gob.mx/siem/estadisticas/ EstadoTamanoPublico.asp?p=1.

Sledgianowski, D., Luftman, J. \& Reilly, R. (2006). Development and validation of an instrument to measure maturity of it business strategic alignment mechanisms. En: Information Resources Management Journal,19: 18-32.

Velázquez, G. (2005). Liderazgo empático: un modelo de liderazgo para las empresas mexicanas. En: Revista del Centro de Investigación, Universidad La Salle, 23(6): 81-100

Wright, L. (2009). The universal qualities of effective leadership. Security Magazine.

Zarate, R. \& Matviuk, S. (2012). Inteligencia emocional y prácticas de liderazgo en las organizaciones colombianas. En: Cuadernos de Administración, 28 (47): 91-103. 